\title{
ON FUNCTIONS THAT ARE TRIVIAL COCYCLES FOR A SET OF IRRATIONALS. II
}

\author{
LAWRENCE W. BAGGETT, HERBERT A. MEDINA, AND KATHY D. MERRILL
}

(Communicated by J. Marshall Ash)

\begin{abstract}
Two results are obtained about the topological size of the set of irrationals for which a given function is a trivial cocycle. An example of a continuous function which is a coboundary with non- $L^{1}$ cobounding function is constructed.
\end{abstract}

A function $v: \mathbb{R} / \mathbb{Z} \rightarrow \mathbb{R}$ is called an (additive) coboundary for an irrational $\alpha$ if there is a measurable function $w: \mathbb{R} / \mathbb{Z} \rightarrow \mathbb{R}$ such that $v(x)=w(x)-w(x+\alpha)$ a.e. (where we parameterize $\mathbb{R} / \mathbb{Z}$ by the interval $[0,1)$ with addition mod 1 ). It is called trivial if $v(x)-c$ is a coboundary for some $c \in \mathbb{R}$. In either case the function $w$, which is unique up to an additive constant, is called the cobounding function. The question of whether particular functions or classes of functions are coboundaries for a given $\alpha$ has applications in ergodic theory and the representation theory of non-Type I groups (see, for example, [BM1],[ILR]). Recent research has revealed an interesting interplay between classes of functions and the types of irrationals for which they can be coboundaries (e.g., [BM2], [M]). Thus it is natural to look at the coboundary question from an opposite point of view, fixing a function $v$, and asking for exactly which irrationals $v$ is a coboundary. A simple Fourier series argument shows that a trigonometric polynomial must be a coboundary for every irrational $\alpha$. For other types of functions, this question is much harder to answer.

In a 1988 paper in this journal [B], L. Baggett presented a proof that the set of irrationals for which a given continuous function is a coboundary must be of the first category, unless the function is a trigonometric polynomial. Shortly after this paper appeared, P. Liardet, A. Iwanik, and P. Hellekalek pointed out a gap in the proof. This gap remains unfilled. In this paper, we present an altered version of that proof in the case that the original function is real-analytic. We also present a parallel result for $L^{1}$ functions in which the cobounding function is also required to be $L^{1}$. Finally, we display a counterexample which shows that the requirement of an $L^{1}$ cobounding function can be a genuine restriction.

Theorem 1. Let $v$ be an integrable, real-analytic function on the open interval $(0,1)$, which is not a trigonometric polynomial. Then the set $S$ of all irrationals for which $v$ is a trivial cocycle is of the first category.

Received by the editors November 15, 1993 and, in revised form, June 21, 1994.

1991 Mathematics Subject Classification. Primary 28D05, 11K38. 
Proof. Suppose $S$ is not of the first category. Let $f(x)=e^{2 \pi i v(x)}$. For each integer $k$ and each positive integer $j$, let $A_{k, j}$ be the set of numbers (rational or irrational) $\alpha$ for which there exists a constant $\lambda$ and a measurable function $g$ such that

(1) $\|g\|_{2} \leq 1$.

(2) $|\lambda|=1$.

(3) $\left|\int_{0}^{1} g(x) e^{2 \pi i k x} d x\right| \geq \frac{1}{j}$.

(4) $f(x) g(x+\alpha)=\lambda g(x)$ for almost all $x \in[0,1)$.

We claim that each set $A_{k, j}$ is a closed set. Let $\left\{\alpha_{n}\right\}$ be a sequence in $A_{k, j}$ converging to $\alpha$. For each $\alpha_{n}$, there exists a constant $\lambda_{n}$ and a measurable function $g_{n}$ satisfying (1)-(4) above. By passing to a subsequence twice, we may assume that $\lambda_{n} \rightarrow \lambda$ and $g_{n} \rightarrow g$ weakly in $L^{2}$. $\lambda$ and $g$ satisfy conditions (1)-(3) above, and a computation shows that $f(x) g(x+\alpha)=\lambda g(x)$ for almost all $x \in[0,1)$. Thus $\alpha \in A_{k, j}$.

Clearly $S \subseteq \bigcup_{k, j} A_{k, j}$. By the Baire Theorem, some set $A_{k_{0}, j_{0}}$ must contain an open interval. Therefore, there exists a positive integer $Q$ such that for every $q \geq Q$ there is a rational number $p / q \in A_{k_{0}, j_{0}}$, and thus a constant $\lambda_{q}$ and a function $g_{q}$ satisfying

(1) $\left\|g_{q}\right\|_{2} \leq 1$.

(2) $\left|\lambda_{q}\right|=1$.

(3) $\left|\int_{0}^{1} g_{q}(x) e^{2 \pi i k_{0} x} d x\right| \geq \frac{1}{j_{0}}$.

(4) $f(x) g_{q}\left(x+\frac{p}{q}\right)=\lambda_{q} g_{q}(x)$ for almost all $x \in[0,1)$.

We also may assume that $p$ is relatively prime to $q$. It follows by condition (4) that for each $q \geq Q$ there exists a $p$ relatively prime to $q$ such that

$$
f(x) f\left(x+\frac{p}{q}\right) f\left(x+\frac{2 p}{q}\right) \ldots f\left(x+\frac{(q-1) p}{q}\right)=\lambda_{q}^{q}
$$

for every $x$ for which $g_{q}(x) \neq 0$, and, by condition (3), this is certainly a set of positive measure.

Now the function

$$
f(x) f\left(x+\frac{p}{q}\right) \ldots f\left(x+\frac{(q-1) p}{q}\right)
$$

has discontinuities at most at the multiples of $\frac{p}{q}$, and on each subinterval $\left(\frac{j}{q}, \frac{(j+1)}{q}\right)$ it is real-analytic. By the identity theorem for real-analytic functions, it follows that

$$
f(x) f\left(x+\frac{p}{q}\right) \ldots f\left(x+\frac{p(q-1)}{q}\right)
$$

is identically $\lambda_{q}^{q}$ on some one of these subintervals. By the invariance of $(*)$ under translation by $\frac{p}{q}$, it follows that

$$
f(x) f\left(x+\frac{p}{q}\right) \ldots f\left(x+\frac{p(q-1)}{q}\right) \equiv \lambda_{q}^{q}
$$

for all $x$ not of the form $\frac{p j}{q}$.

Now $f(x)=e^{2 \pi i v(x)}$, so we have that

$$
v(x)+v\left(x+\frac{p}{q}\right)+\ldots+v\left(x+\frac{p(q-1)}{q}\right)=c_{q}+N_{q}(x)
$$


where $c_{q}$ is a constant and $N_{q}$ is an integer-valued function. Because $v$ is continuous, we have that $N_{q}$ is constant on the subintervals $\left(\frac{j}{q}, \frac{(j+1)}{q}\right)$.

Using (**), we compute the $n q$ th Fourier coefficient of $v, c_{n q}(v)$, and obtain

$$
q c_{n q}(v)=0
$$

for every nonzero integer $n$. Since this computation holds for every $q \geq Q$, it follows immediately that $v$ is a trigonometric polynomial.

Remark. Michael Herman [H, Theorem 4.11] proved a similar result under the additional hypothesis that for all $n \neq 0, c_{n}(v) \neq 0$.

By requiring the cobounding functions to be integrable, we obtain the following stronger result.

Theorem 2. Let $v$ be a real-valued $L^{1}$ function on $\mathbb{R} / \mathbb{Z}$, which is not a trigonometric polynomial. Then the set of all irrationals for which $v$ is a trivial cocycle with $L^{1}$ cobounding function is of the first category.

Proof. By the Riemann-Lebesgue Lemma, if $v$ is a coboundary for $\alpha$ with $L^{1}$ cobounding function $w$, then $\left|c_{n}(w)\right|=\left|c_{n}(v)\right| /\left|1-e^{2 \pi i n \alpha}\right| \rightarrow 0$ as $|n| \rightarrow \infty$. Thus it will suffice to find a dense $G_{\delta}$ set $E$ of irrationals such that for $\alpha \in E$, $\left|c_{n}(v)\right| /\left|1-e^{2 \pi i n \alpha}\right| \nrightarrow 0$. Since $v$ is not a trigonometric polynomial, $\exists\left\{m_{k}\right\}_{k=1}^{\infty}$, $m_{k} \rightarrow \infty$, such that $\left|c_{m_{k}}(v)\right|=\epsilon_{k} \neq 0$. Choose $a_{k}$ so that $a_{k}>\frac{1}{m_{k} \epsilon_{k}}$. Let

$$
A_{k}=\bigcup_{j=1}^{m_{k}-1}\left(\frac{j}{m_{k}}-\frac{1}{a_{k} m_{k}^{2}}, \frac{j}{m_{k}}+\frac{1}{a_{k} m_{k}^{2}}\right) .
$$

If $\alpha \in A_{k}$, then $\exists j$ such that $\left|\alpha-\frac{j}{m_{k}}\right|<\frac{1}{a_{k} m_{k}^{2}}$, which implies $\left|m_{k} \alpha-j\right|<\frac{1}{a_{k} m_{k}}$ and hence $\left|1-e^{2 \pi i m_{k} \alpha}\right|<\frac{1}{a_{k} m_{k}}$. Thus we see that for $\alpha \in A_{k},\left|c_{m_{k}}(v)\right| /\left|1-e^{2 \pi i m_{k} \alpha}\right|>1$. Let $E_{n}=\bigcup_{k=n}^{\infty} A_{k}$. $E_{n}$ is open and dense for each $n$, and by the above we have that if $\alpha \in E_{n}, \exists m_{k}, k \geq n$, such that $\left|c_{m_{k}}(v)\right| /\left|1-e^{2 \pi i m_{k} \alpha}\right|>1$. Set $E=\bigcap_{n=1}^{\infty} E_{n}$.

The apparent advantage of the second theorem over the first raises the natural question of whether an $L^{1}$ coboundary, or even an analytic coboundary, must have an $L^{1}$ cobounding function.

Theorem 3. Given any irrational $\alpha$, there exists a continuous coboundary $v$ for $\alpha$, whose cobounding function is not $L^{1}$.

Proof. Choose a sequence of rationals $\left\{\frac{p_{n}}{q_{n}}\right\}$ satisfying

$$
\left|\alpha-\frac{p_{n}}{q_{n}}\right| \leq \frac{1}{n^{3} 2^{2 n+1} q_{n}} .
$$

(This can be done by choosing a subsequence of the convergents to $\alpha$ so that each element, $\frac{p_{n}}{q_{n}}$, of this subsequence has the property that $q_{n} \geq n^{3} 2^{2 n+1}$.) For each $n \geq 1$, we define the function $u_{n}$ by

$$
u_{n}(x)= \begin{cases}2^{n+1}+n 2^{2 n+1} q_{n} x & \text { if } x \in\left(-\frac{1}{n 2^{n} q_{n}}, 0\right), \\ 2^{n+1}-n 2^{2 n+1} q_{n} x & \text { if } x \in\left(0, \frac{1}{n 2^{2} q_{n}}\right),\end{cases}
$$


and then define

$$
w_{n}(x)=\sum_{p=0}^{q_{n}-1} u_{n}\left(x-\frac{p}{q_{n}}\right) .
$$

(The function $w_{n}$ is triangular on $\left(\frac{p}{q_{n}}-\frac{1}{n 2^{n} q_{n}}, \frac{p}{q_{n}}+\frac{1}{n 2^{n} q_{n}}\right)$ with $w_{n}\left(\frac{p}{q_{n}}\right)=2^{n+1}$ for $p=0,1, \ldots, q_{n}-1$, and 0 everywhere else.) Finally, let

$$
w(x)=\sum_{n=1}^{\infty} w_{n}(x)
$$

To show that $w$ is finite a.e., we show that $S_{N}=\sum_{n=1}^{N} w_{n}$ is Cauchy in measure. Indeed, for any $N>M, S_{N}-S_{M}=\sum_{n=M+1}^{N} w_{n}$ is supported on a set of measure $\sum_{n=M+1}^{N}\left(q_{n}\right)\left(\frac{1}{n 2^{n} q_{n}}\right)$, which goes to zero as $N$ and $M$ go to infinity. We see that $w$ is not in $L^{1}$ by noting that $\int\left|w_{n}(x)\right| d x=\frac{2}{n}$ so that by the monotone convergence theorem we have

$$
\int|w(x)| d x=\sum_{n=1}^{\infty} \int\left|w_{n}(x)\right| d x=\sum_{n=1}^{\infty} \frac{2}{n}=\infty .
$$

Now we define

$$
v(x)=w(x)-w(x+\alpha)=\sum_{n=1}^{\infty} w_{n}(x)-w_{n}(x+\alpha) .
$$

Since the $w_{n}(x)-w_{n}(x+\alpha)$ are continuous, it will follow from the M-test that $v$ is continuous, if we can show that $\left|w_{n}(x)-w_{n}(x+\alpha)\right|<\frac{1}{n^{2}}$. By the periodicity of $w_{n}$, we have that

$$
\left|w_{n}(x)-w_{n}(x+\alpha)\right|=\left|w_{n}\left(x+\frac{p_{n}}{q_{n}}\right)-w_{n}(x+\alpha)\right| \leq n 2^{2 n+1} q_{n}\left|\alpha-\frac{p_{n}}{q_{n}}\right|<\frac{1}{n^{2}},
$$

since $n 2^{2 n+1} q_{n}$ is the maximum slope of a secant line of $w_{n}$.

Remark. For certain $\alpha$, we can modify the above construction to give $C^{r}$ coboundaries with non- $L^{1}$ cobounding functions. In particular, if there is a sequence of rational approximations to $\alpha,\left\{\frac{p_{n}}{q_{n}}\right\}$, such that $\left|\alpha-\frac{p_{n}}{q_{n}}\right|<\frac{1}{q_{n}^{r+2}}$, we can replace the continuous, piecewise linear functions $w_{n}$ with $C^{r}$, piecewise $(r+1)$ st degree polynomials, with the same integral as before, and with the property that $\sum w_{n}^{(r)}(x)-w_{n}^{(r)}(x+\alpha)$ converges uniformly, thus giving $v$ a continuous $r$ th derivative. Y. Meyer [H, p. 187] has a related result in the $r=1$ case, which implies that if $\alpha$ has bounded partial quotients in its continued fraction expansion, then there exists a $C^{1}$ function which is a coboundary for $\alpha$ with noncontinuous cobounding function. The question of whether there are analytic coboundaries with non- $L^{1}$ cobounding functions remains unanswered. 


\section{REFERENCES}

[B] L. Baggett, On functions that are trivial for a set of irrationals, Proc. Amer. Math. Soc. 104 (1988), 1212-1215. MR 89h:28022a

[BM1] L. Baggett and K. Merrill, Representations of the Mautner group and cocycles of an irrational rotation, Michigan Math. J. 33 (1986), 221-229. MR 87h:22011

[BM2] _ Smooth cocycles for an irrational rotation, Israel J. Math. 79 (1992), 281-288. MR 95e: 28017

[H] M. Herman, Sur la conjugaison différentiable des difféomorphismes du cercle à des rotations, Inst. Hautes Études Sci. Publ. Math. 49 (1979), 5-234. MR 81h:58039

[ILR] A. Iwanik, M. Lemańczyk, and D. Rudolph, Absolutely continuous cocycles over irrational rotations, Israel J. Math. 83 (1993), 73-95. MR 94i:58108

[M] H. Medina, Spectral types of unitary operators arising from irrational rotations on the circle group, Michigan Math. J. 41 (1994), 39-49. MR 95a:28014

Department of Mathematics, University of Colorado, Boulder, Colorado 80309

E-mail address: baggett@euclid.colorado.edu

Department of Mathematics, Loyola Marymount University, Los Angeles, California 90045

E-mail address: hmedina@lmumail.lmu.edu

Department of Mathematics, The Colorado College, Colorado Springs, Colorado 80903

E-mail address: kmerrill@cc.colorado.edu 